Supporting Information

\title{
Revealing the Structural Aspect of Ultrastable Self- Supportive Bifunctional Electrocatalyst for Solar-Driven Water Splitting
}

Manisha Das, Navpreet Kamboj, Taniya Purkait, Subhajit Sarkar, Ramendra Sundar Dey*

Institute of Nano Science and Technology, Phase-10, Sector-64, Mohali, Punjab 160062, India.

*E-mail: rsdey@inst.ac.in 


\section{Experimental section}

\section{Materials}

All of the chemicals and reagents used in the experiment are of analytically pure grade and used as received without further purification. Nickel chloride hexahydrate $\left(\mathrm{NiCl}_{2} \cdot 6 \mathrm{H}_{2} \mathrm{O}\right)$, sodium dihydrogen phosphate $\left(\mathrm{NaH}_{2} \mathrm{PO}_{4} \cdot \mathrm{H}_{2} \mathrm{O}\right)$, nitric acid $\left(\mathrm{HNO}_{3}\right)$, sulphuric acid $\left(\mathrm{H}_{2} \mathrm{SO}_{4}\right)$, copper sulphate $\left(\mathrm{CuSO}_{4} \cdot 5 \mathrm{H}_{2} \mathrm{O}\right)$, sodium hypophosphite $\left(\mathrm{NaH}_{2} \mathrm{PO}_{2} \cdot \mathrm{H}_{2} \mathrm{O}\right)$, graphite powder, graphite plate, Highly pure graphite flakes $(98 \%)$, platinum on carbon $(\mathrm{Pt} / \mathrm{C})$, and $\mathrm{RuO}_{2}$ were purchased from Sigma Aldrich. And Hydrochloric acid $(\mathrm{HCl})$ was purchased from Merck chemicals India. Copper foil with high purity was purchased from Gelon LIB group, China (Thickness $(\mathrm{d})=1-5 \mu \mathrm{m})$.

\section{Preparation of copper foam electrode as a substrate}

A 3-dimensional dendritic foam of copper was grown on copper foil in a two-electrode process by galvanostatic electrodeposition method based on our already reported method with few modifications. A piece of copper foil $\left(1 \times 1 \mathrm{~cm}^{2}\right)$ was cleaned with $30 \%$ dilute nitric acid, deionized (DI) water and finally with ethanol. The washed copper foil was used as both working (cathode) and counter (anode) electrode and kept at a distance of $\sim 1.5 \mathrm{~cm}$. A constant current density of $2 \mathrm{~A} \mathrm{~cm}^{-2}$ was applied between the electrodes placed in an electrochemical cell containing $0.4 \mathrm{M} \mathrm{CuSO}_{4}$ in $1.5 \mathrm{M} \mathrm{H}_{2} \mathrm{SO}_{4}$ for a duration of $45 \mathrm{~s}$. As fabricated Cuf was then washed properly with DI water to remove any present acidic residue.

\section{$\mathrm{Pt} / \mathrm{C}$ and $\mathrm{RuO}_{2}$ fabrication}

An amount of $5 \mathrm{mg}$ of the commercially available $\mathrm{Pt} / \mathrm{C}$ and $\mathrm{RuO}_{2}$ were taken and dispersed in a $1 \mathrm{~mL}$ mixed solution of water and ethanol (volume ratio $=1: 1)$ and sonicated for at least 30 min to generate a homogeneous ink. After that both $\mathrm{Pt} / \mathrm{C}$ and $\mathrm{RuO}_{2}$ inks ware further dropcasted onto two separate glassy carbon electrodes $\left(0.07 \mathrm{~cm}^{2}\right)$ with a mass loading of 0.5 $\mathrm{mg} \mathrm{cm}{ }^{-2}$. 


\section{Physical Characterizations}

Electrochemical measurements performed in this experiment were carried out with CHI 760E electrochemical workstation and Metrohm Autolab (M204 multichannel potentiostat galvanostat) attached with FRA 32M module. X-ray diffraction (XRD) pattern was obtained on a Bruker D8 Advances instrument using $\mathrm{Cu}-\mathrm{K} \alpha(\lambda=1.5406 \AA)$ radiation with an acceleration voltage of $40 \mathrm{KV}$ in the $2 \theta$ range from $10^{\circ}$ to $80^{\circ}$. For the investigation the surface morphology and the elemental analysis of the synthesized material, Scanning Electron Microscopy (SEM) JeolJSMIT300 equipped with a BrukerXFlash 6130 integrated with Energy Dispersive X-ray Spectroscopy (EDS) were performed. JEM2100 were utilized to perform Transmission Electron Microscopy (TEM), High-Resolution TEM (HRTEM), Selected Area Electron Diffraction (SAED) and elemental mapping studies. X-ray photoelectron spectroscopy (XPS) with an ultrahigh vacuum chamber $\left(2 \times 10^{-9}\right.$ mbar $)$ using (Monchromatic) with $6 \mathrm{~mA}$ beam current by K $\alpha$ plus XPS system by ThermoFisher Scientific instruments (UK) were recorded to know the chemical states of the sample. Drop size analyzer, KRUSS (DSA25E, 100 watt) was used to know the hydrophilic nature of the catalyst. Raman spectra were obtained in a WITEC Focus Innovations Alpha-300 Raman confocal microscope with an excitation laser wavelength of $532 \mathrm{~nm}$. For the realization of hybridization of different units with solar cell, a mini solar panel of $6 \mathrm{~V}$ was used. A $400 \mathrm{~W}$ Xenon lamp (ORIEL Instruments; OPS-A1000) with Newport (FSQGG400) filter was used to illuminate the solar panel. For testing the purity of evolved gas during electrolysis, Gas chromatography experiment were performed with the help of PerkinElmer, Arnel Engineered Solutions, Clarus 680 GC.

For the analysis of bodings and chemical states, XANES spectra were recorded at BL9, Scanning EXAFS Beamline of Indus-2. The measurements were done in transmission mode. The beamline consisted of $\mathrm{Rh} / \mathrm{Pt}$ coated meridional cylindrical mirror for collimation 
and a $\mathrm{Si}(111)$ double crystal monochromator (DCM) to select excitation energy of $\mathrm{Ni}$ and $\mathrm{Cu}$ K-edges. The second crystal of the DCM is a saggital cylinder which provided beam focused in horizontal direction.

\section{Catalyst surface property (Hydrophilicity and Aerophobicity)}

Surface property of the catalyst is very important parameter in catalysis. The proper contact between the electrode and the electrolyte is crucial in electrolysis of water. Thus, the catalyst must be superhydrophilic in order to promote the activity of the catalyst. We have performed the contact angle of the all the catalysts including control catalysts with a drop of water. Fig. 5a shows the surface of Cuf@ $\mathrm{Ni}_{5} \mathrm{P}_{4}$ when the drop was about to fall on surface, as soon as the drop falls on the surface it gets absorbed (Fig. 5b) within the fraction of second with $0^{\circ}$ contact angle, proves that the catalyst is highly hydrophilic in nature. The hydrophilic study of bare Cuf (Fig. S13a, Supporting Information), $\mathrm{Ni}_{5} \mathrm{P}_{4} / \mathrm{Cu}$ (Fig. S13b, Supporting Information) and $\mathrm{Ni}_{5} \mathrm{P}_{4} / \mathrm{GP}$ (S13c, Supporting Information) were also performed. Clearly, bare Cuf proved to be the super hydrophilic surface but other two i.e. $\mathrm{Ni}_{5} \mathrm{P}_{4} / \mathrm{Cu}$ and $\mathrm{Ni}_{5} \mathrm{P}_{4} / \mathrm{GP}$ imposed a contact angle of $89^{\circ}$ and $20^{\circ}$, respectively. The renewal and removal of gas bubbles from the surface of the catalyst during electrolysis is very critical in order to avoid its adhesion on the surface that blocks and fluctuate the active surface area and thus degrades the catalytic activity. The vertically aligned, dendritic and porous structure of core shell nanostructure of Cuf@ $\mathrm{Ni}_{5} \mathrm{P}_{4}$, enables it for the self-removal of as formed $\mathrm{H}_{2}$ and $\mathrm{O}_{2}$ gas bubbles before it grows large and engage the surface. As shown in Fig. 5c-f, the bubble starts to grow from $100 \mu \mathrm{m}$ (Fig. 5d) to a maximum of $150 \mu \mathrm{m}$ (Fig. 5e) and leaves surface as soon as it grows further at varying CP time and a very few bubbles were observed on the surface of the catalyst when the CP stopped (Fig. 5f). This fast renewal and release of the bubble avoid to cause the catalyst degradation and maintains a constant area for catalytic activity. The schematic representation for the possible mechanism of bubble formation is shown in Fig. $5 \mathrm{~g}$. 
For more clear explanation and understanding, an experiment for the bubble formation and releasing pattern on bare $\mathrm{Cuf}$ (Fig. S13a-c, Supporting Information), $\mathrm{Ni}_{5} \mathrm{P}_{4} / \mathrm{Cu}$ (Fig. S13d and f, Supporting Information) and $\mathrm{Ni}_{5} \mathrm{P}_{4} / \mathrm{GP}$ (Fig. S13g-i, Supporting Information) were also performed and recorded digitally. As shown in Fig. S12 the bubble stays and adhere on the surface strongly and makes it difficult to detach until it grows large enough and thus hinders the kinetics of the catalyst results in corrupting the activity of the catalyst. The buoyancy force dominates on adhesive force when the size of the bubble is large and thus make bubbles to stay on the surface. The small bubbles continued to grow until it became large as in the case of bare Cuf, the bubbles average size reaches a maximum of $\sim 700 \mu \mathrm{m}$, while for $\mathrm{Ni}_{5} \mathrm{P}_{4} / \mathrm{Cu}$ and $\mathrm{Ni}_{5} \mathrm{P}_{4} / \mathrm{GP}$ an average size of gas bubble were $\sim 300 \mu \mathrm{m}$ and $\sim 400 \mu \mathrm{m}$, respectively before it leaves the surface. Thus, Cuf@ $@ \mathrm{Ni}_{5} \mathrm{P}_{4}$ demonstrated such a catalytic surface which leads to rapid and quick removal of gas bubbles from the surface. 


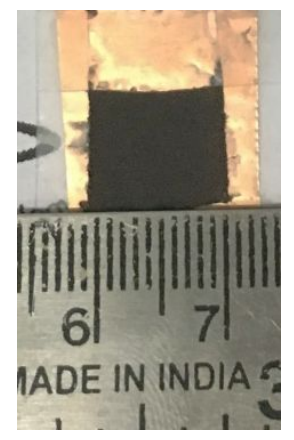

Figure S1. Digital photograph of Cuf@ $\mathrm{Ni}_{5} \mathrm{P}_{4}$ electrode. The deposited electrode area was $1 \times 1 \mathrm{~cm}^{2}$. 

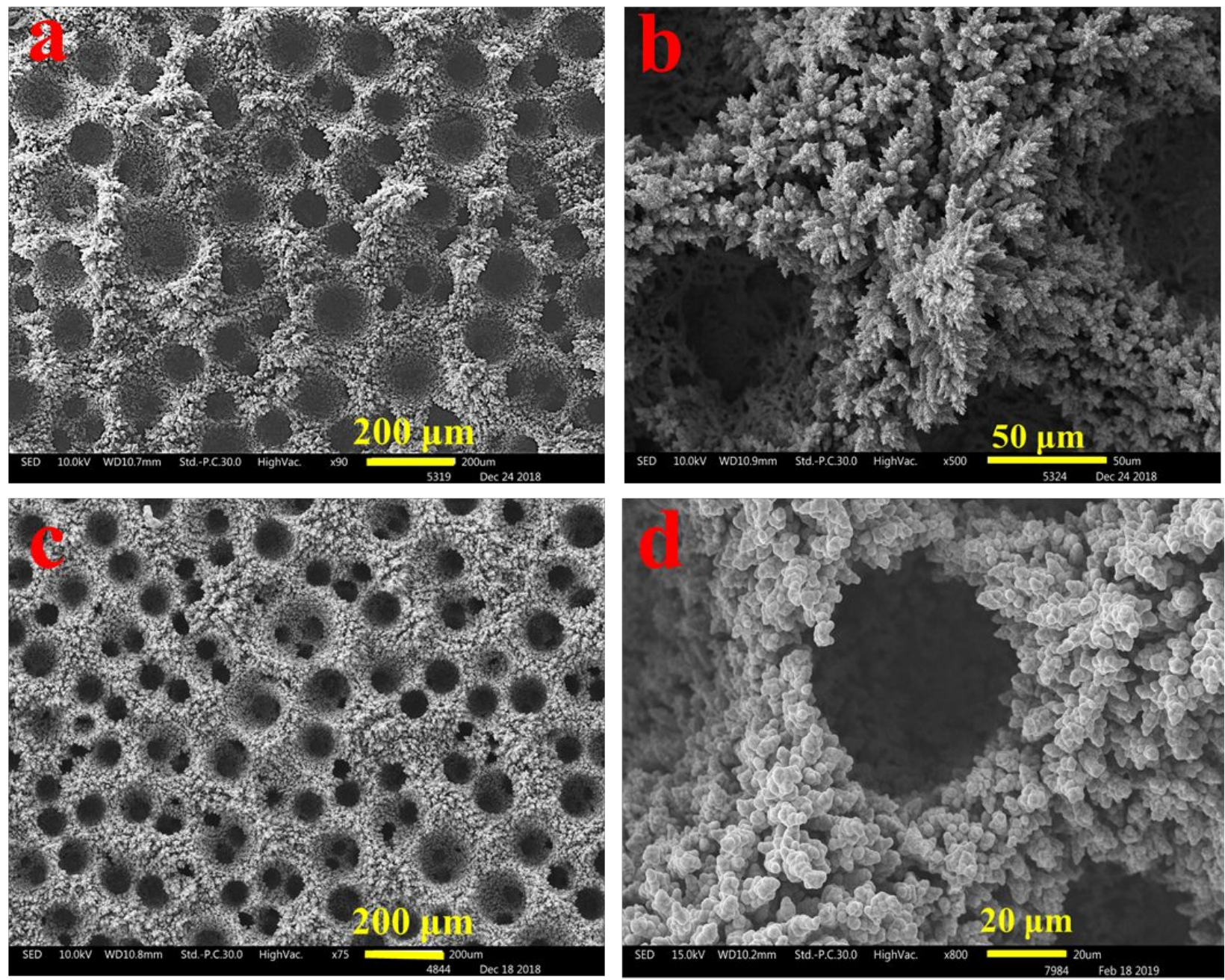

Figure S2. SEM images at different magnifications (a-b) bare Cuf (c-d) Cuf@ $\mathrm{Ni}_{5} \mathrm{P}_{4}$. 

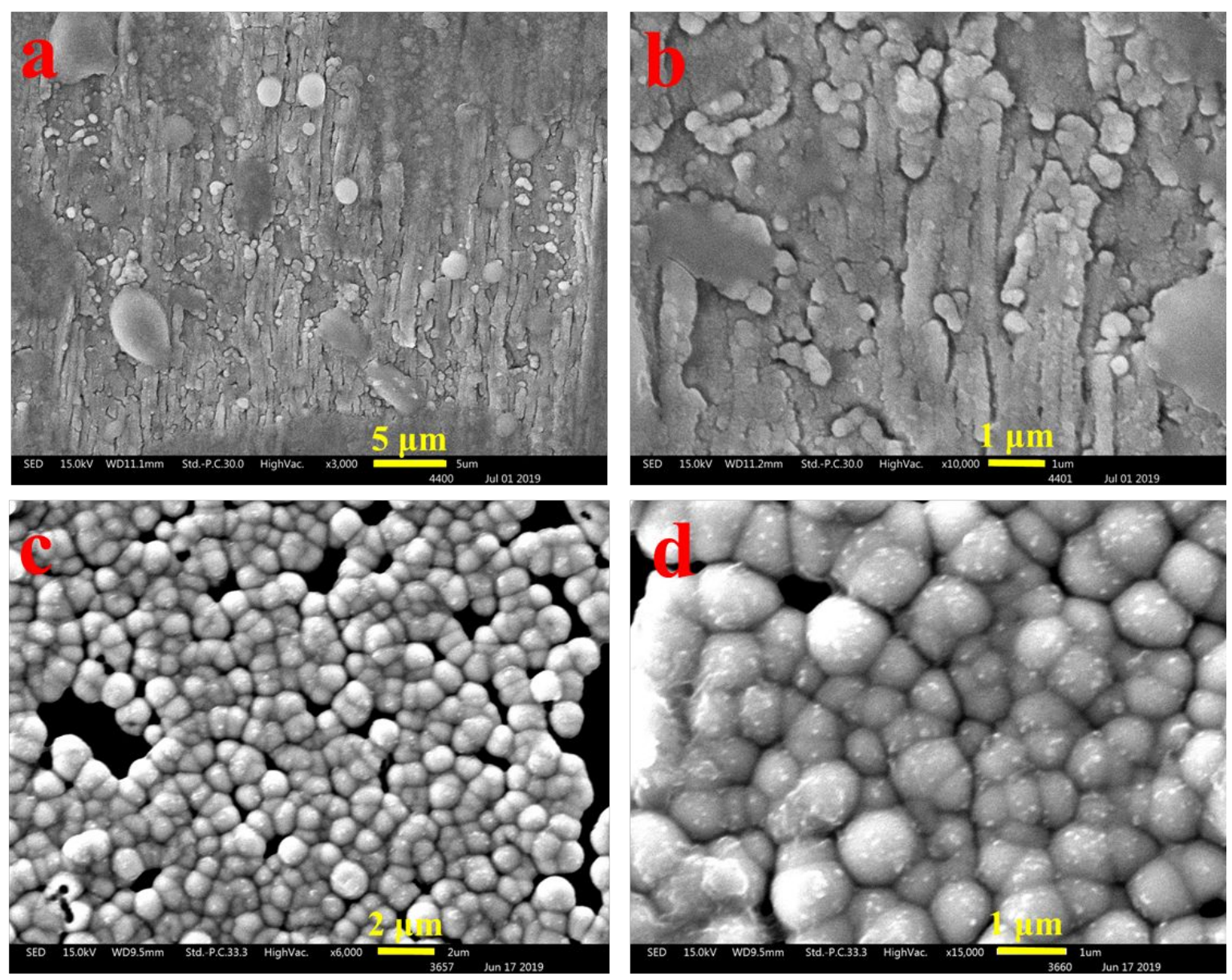

Figure S3. SEM images of $\mathrm{Ni}_{5} \mathrm{P}_{4}$ on different substrates (a-b) $\mathrm{Ni}_{5} \mathrm{P}_{4} / \mathrm{Cu}$ (c-d) $\mathrm{Ni}_{5} \mathrm{P}_{4} / G P$. 

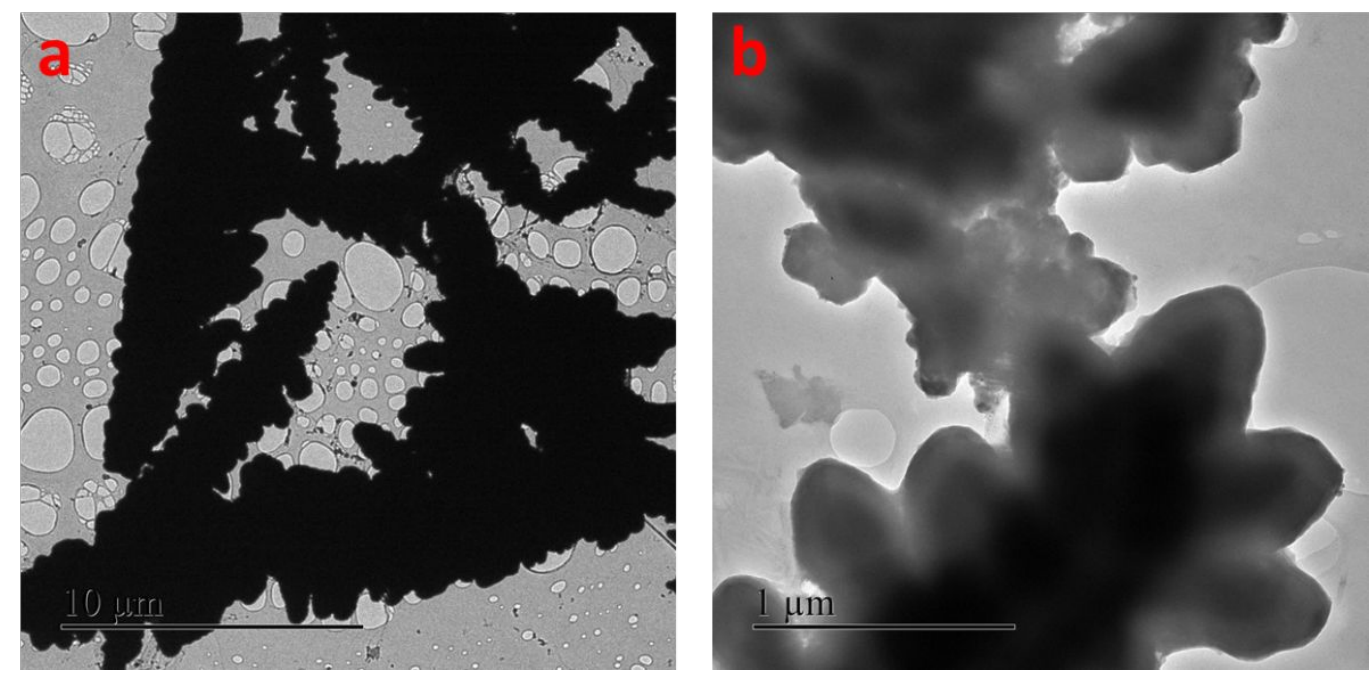

Figure S4. TEM images of Cuf@ $\mathrm{Ni}_{5} \mathrm{P}_{4}$ at different magnifications with a scale bar of (a) $10 \mu \mathrm{m}$ (b) $1 \mu \mathrm{m}$. 


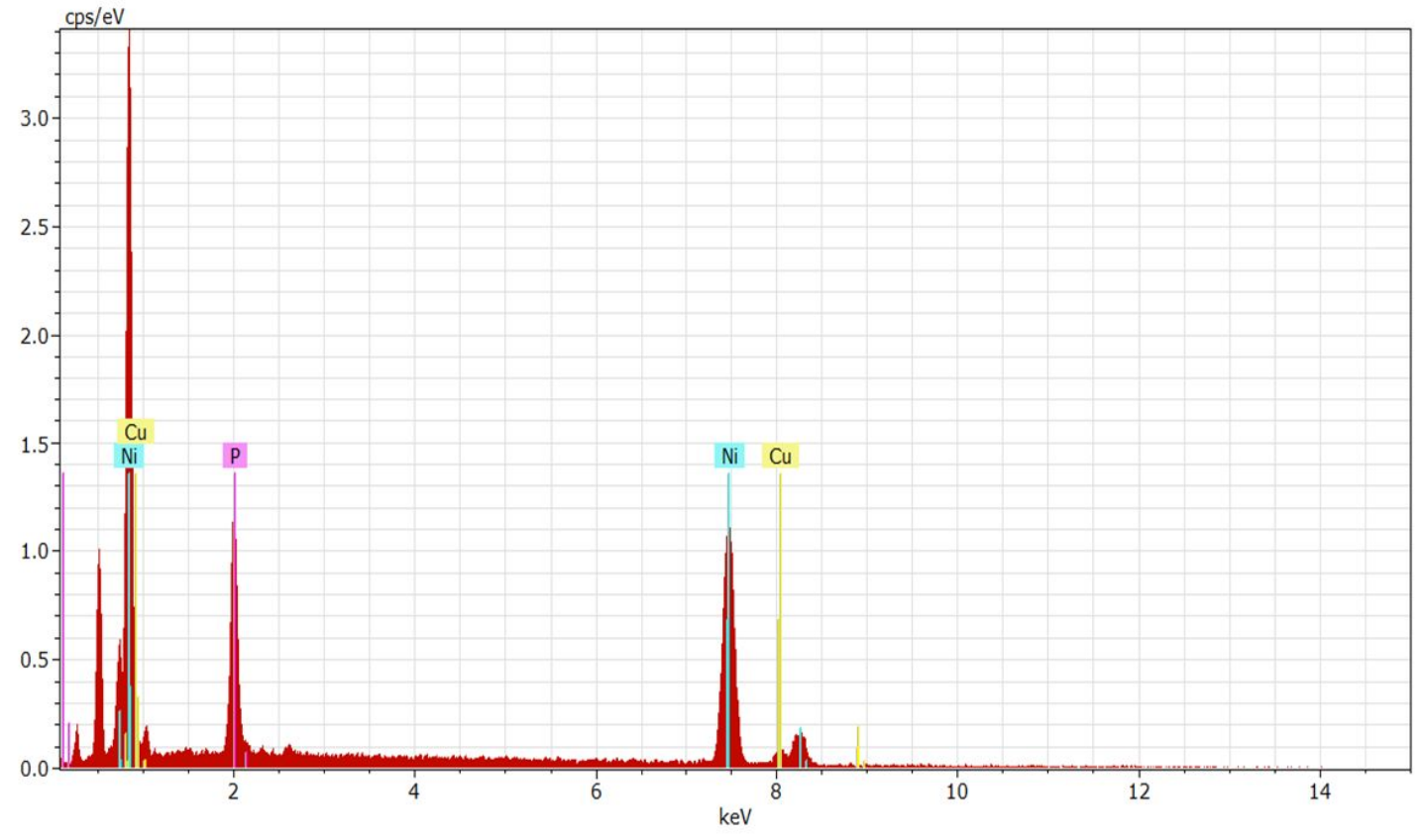

Figure S5. Energy Dispersive X-ray spectroscopic image for Cuf@ $\mathrm{Ni}_{5} \mathrm{P}_{4}$ 

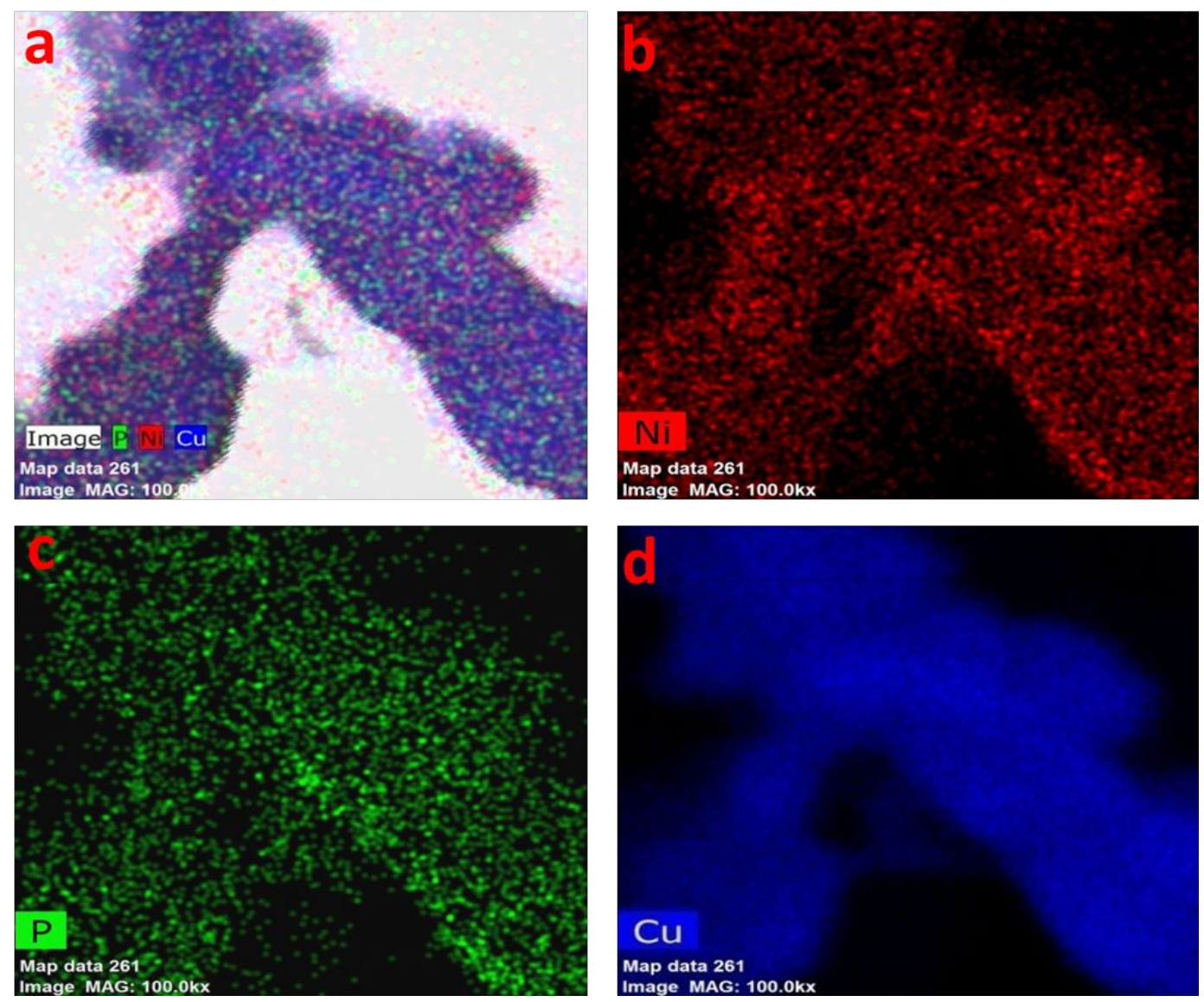

Figure S6. (a-d) Elemental mapping of Cuf@ $\mathrm{Ni}_{5} \mathrm{P}_{4}$ examined from TEM 

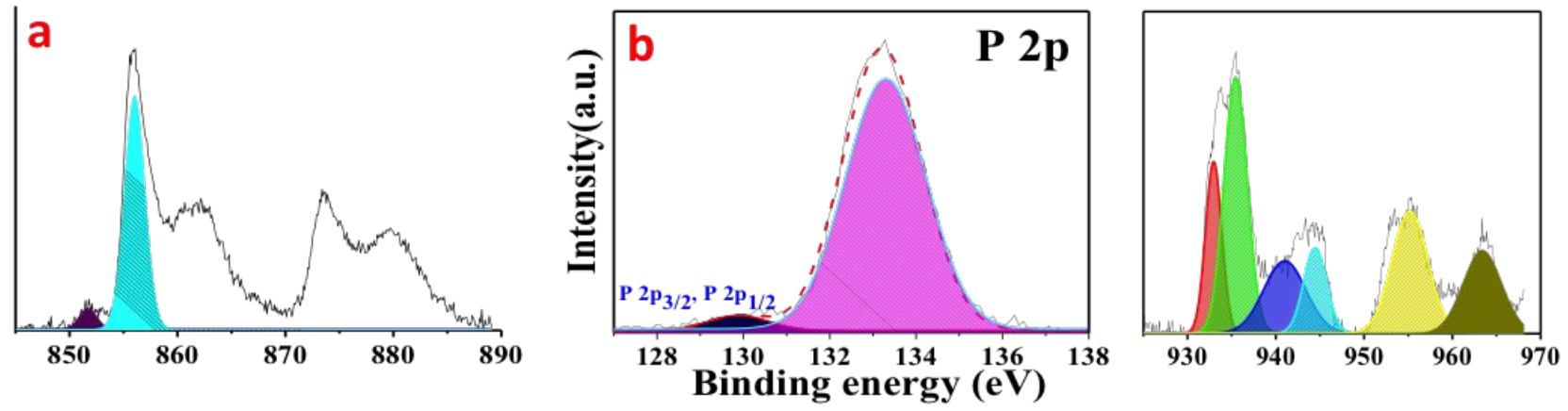

Figure S7. XPS spectra of Cuf@Nis $\mathrm{P}_{4}$ (a) Ni 2p and (b) P 2p (c) Cu 2p 


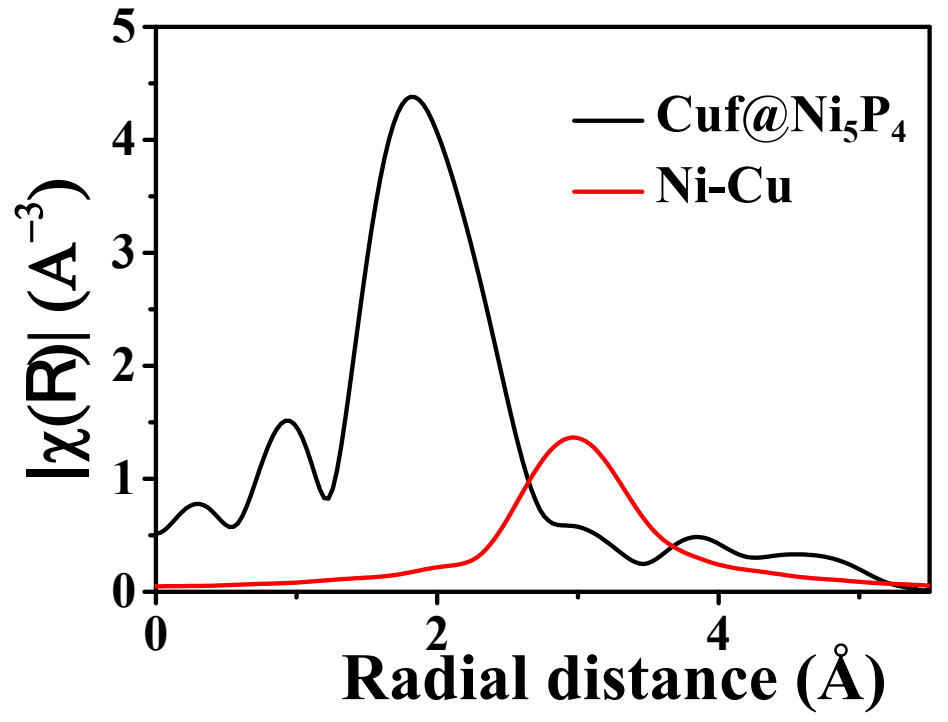

Figure S8. FT-XFAS fitting of $\mathrm{Ni}-\mathrm{Cu}$ bond present in Cuf@ $\mathrm{Ni}_{5} \mathrm{P}_{4}$ 

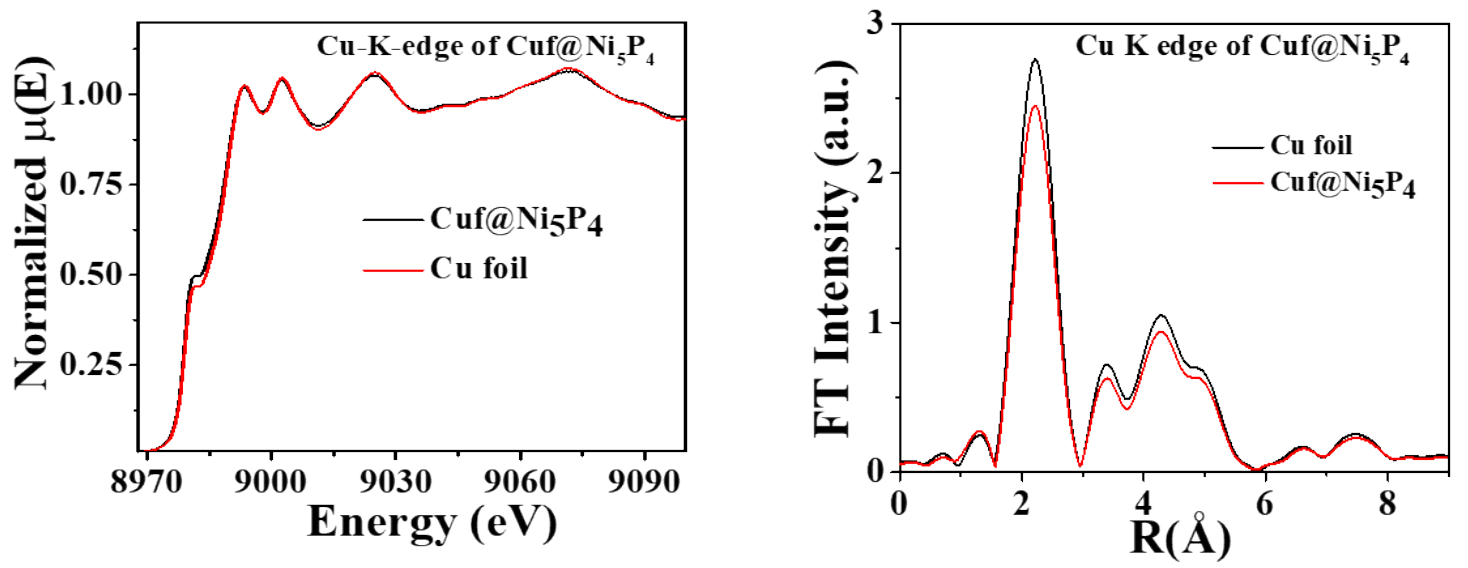

Figure S9. (a) XANES spectra of the catalyst Cuf@ $\mathrm{Ni}_{5} \mathrm{P}_{4}$ with $\mathrm{Cu}$ foil as a reference for Cu K-edge. (b) FT-EXFAS spectra of Cuf@Ni $\mathrm{N}_{4}$ for Cu K-edge. 


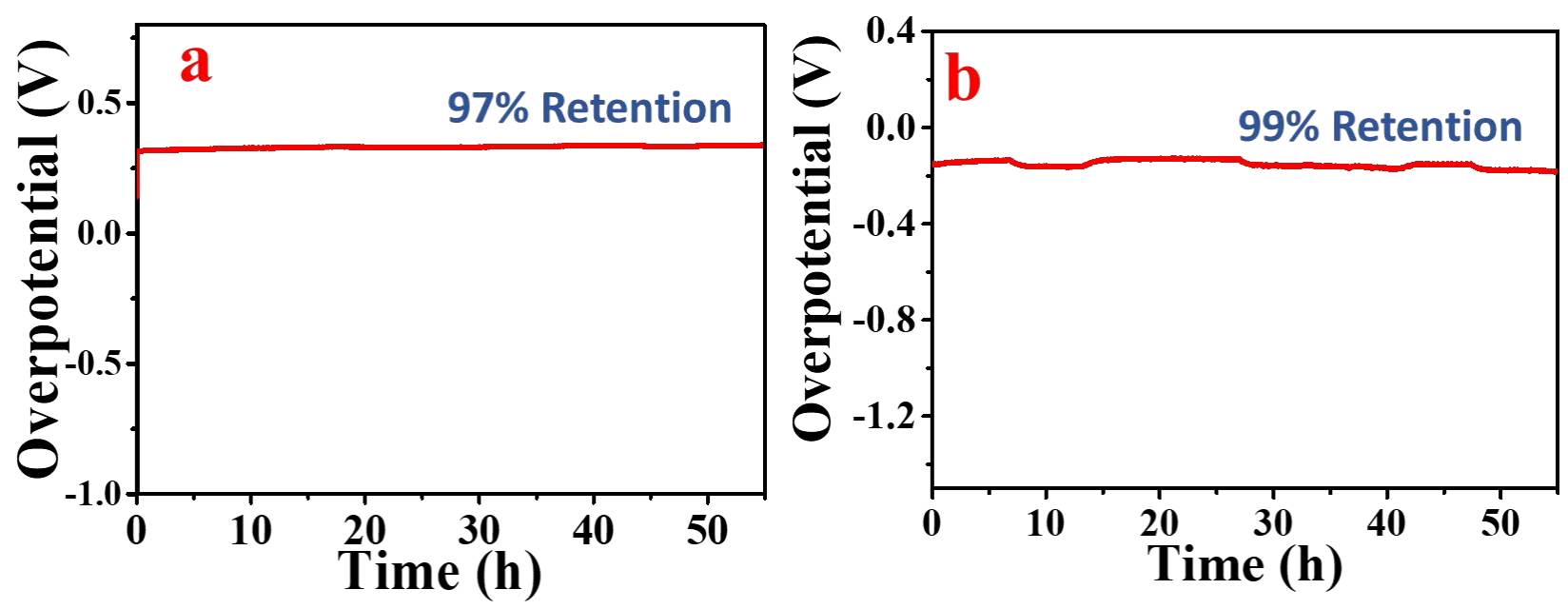

Figure S10. (a) Chronopotentiometric curve of Cuf@Ni ${ }_{5} \mathrm{P}_{4}$ for OER at $J=100 \mathrm{~mA} \mathrm{~cm}^{-2}$ (b) for $\mathrm{HER}$ at $J=10 \mathrm{~mA} \mathrm{~cm}^{-2}$. Experiments were carried out in $1 \mathrm{M} \mathrm{KOH}$. 

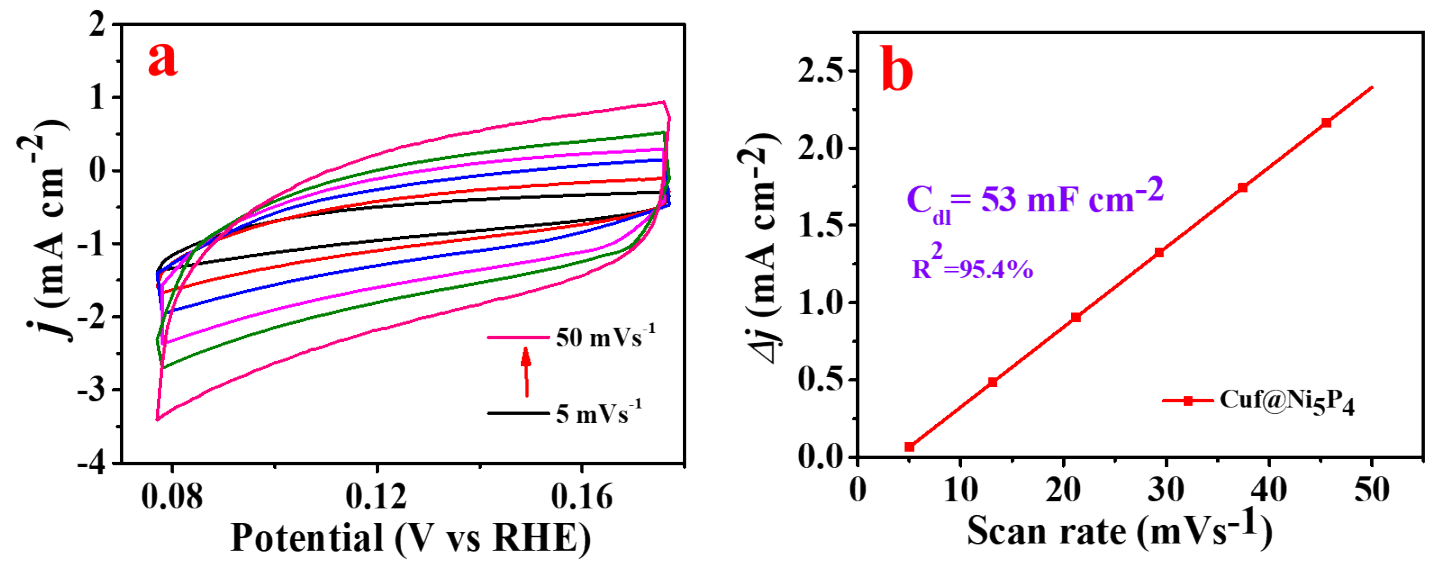

Figure S11. (a) Cyclic voltammetry Cuf@ $\mathrm{Ni}_{5} \mathrm{P}_{4}$ at different scan rates in nonfaradic region (b) Linear fits of current densities at different scan rates of $\mathrm{Cuf} @ \mathrm{Ni}_{5} \mathrm{P}_{4}$ 
(a) Before stability

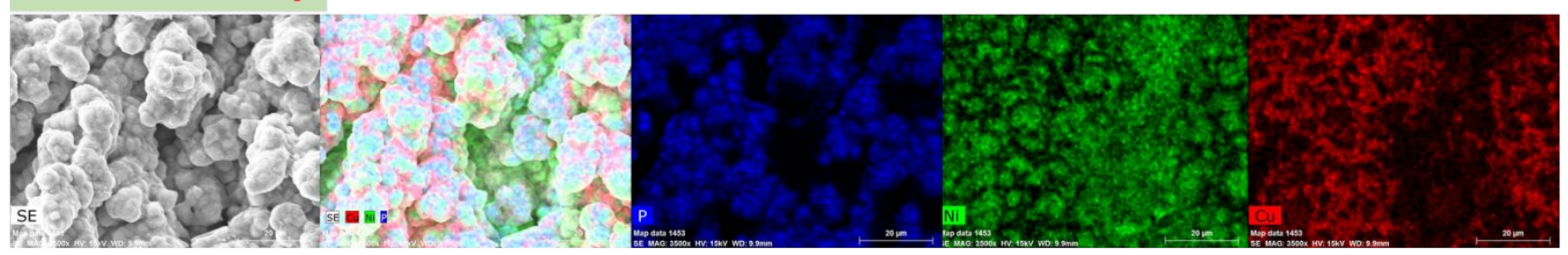

(b) After stability

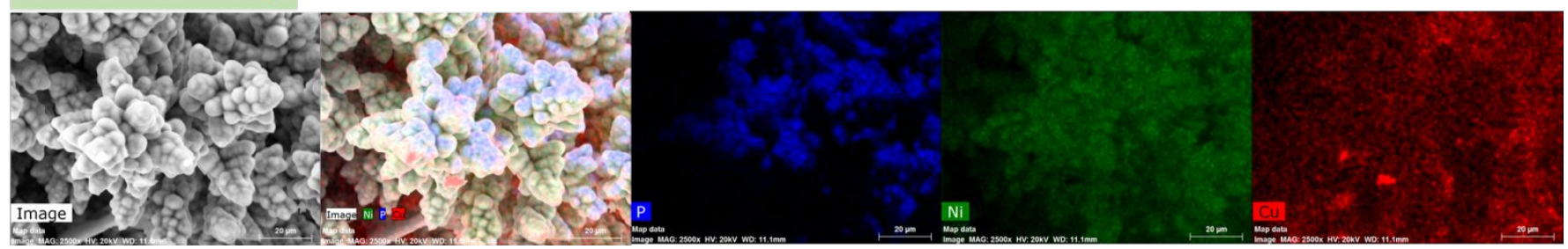

(C) Spectrum: Acquisition 7676.spx

Bl AN Series unn. C norm. C Atom. C Error (1 Sigma) [wt. * ] [wt. * ] [at. * ] [wt.* ]

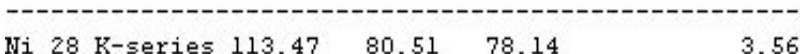

$\begin{array}{lllll}\text { Cu } 29 \mathrm{~K} \text {-series } & 20.90 & 14.83 & 13.30 & 0.78\end{array}$

$\begin{array}{lllll}\text { p } 15 \mathrm{~K} \text {-series } & 6.56 & 4.66 & 8.56 & 0.29\end{array}$

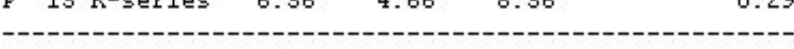

Total: $140.93 \quad 100.00 \quad 100.00$

Spectrum: Acquisition 11275.spx

B1 AN Series unn. C norm. C Atom. C Error (1 Sigma)

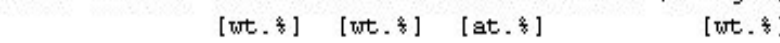

Ni $28 \mathrm{~K}$-series $107.50 \quad 68.87 \quad 68.66 \quad 3.81$

$\begin{array}{lrrrr}\text { Cu } 29 \text { K-series } & 44.28 & 28.37 & 26.12 & 2.02\end{array}$

$\begin{array}{llllll}\text { p } 15 & \text { K-series } & 4.31 & 2.76 & 5.21 & 0.27\end{array}$

P.

Total: $156.09 \quad 100.00 \quad 100.00$

Before stability

After stability

Figure S12. Elemental mapping of Cuf@ $\mathrm{Ni}_{5} \mathrm{P}_{4}$ for elements: P (Blue), Ni (Green), $\mathrm{Cu}$ (Red) (a) before and (b) after stability test through chronopotentiometry. (c) The quantifications of elements obtained before and after the stability study. 


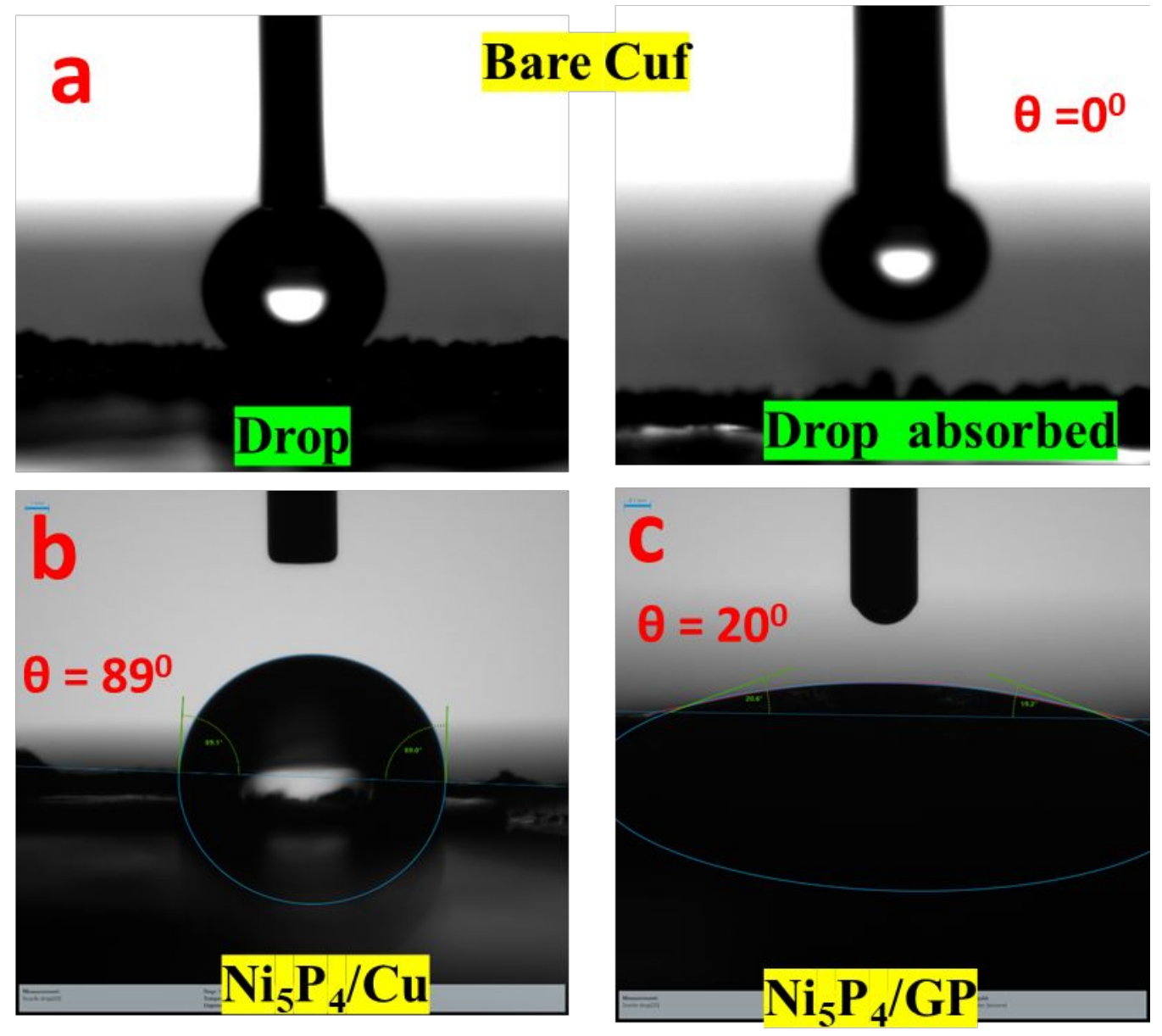

Figure S13. Hydrophilicity analysis of the control samples (a) Bare Cuf (b) $\mathrm{Ni}_{5} \mathrm{P}_{4} / \mathrm{Cu}$ (c) $\mathrm{Ni}_{5} \mathrm{P}_{4} / \mathrm{GP}$ 

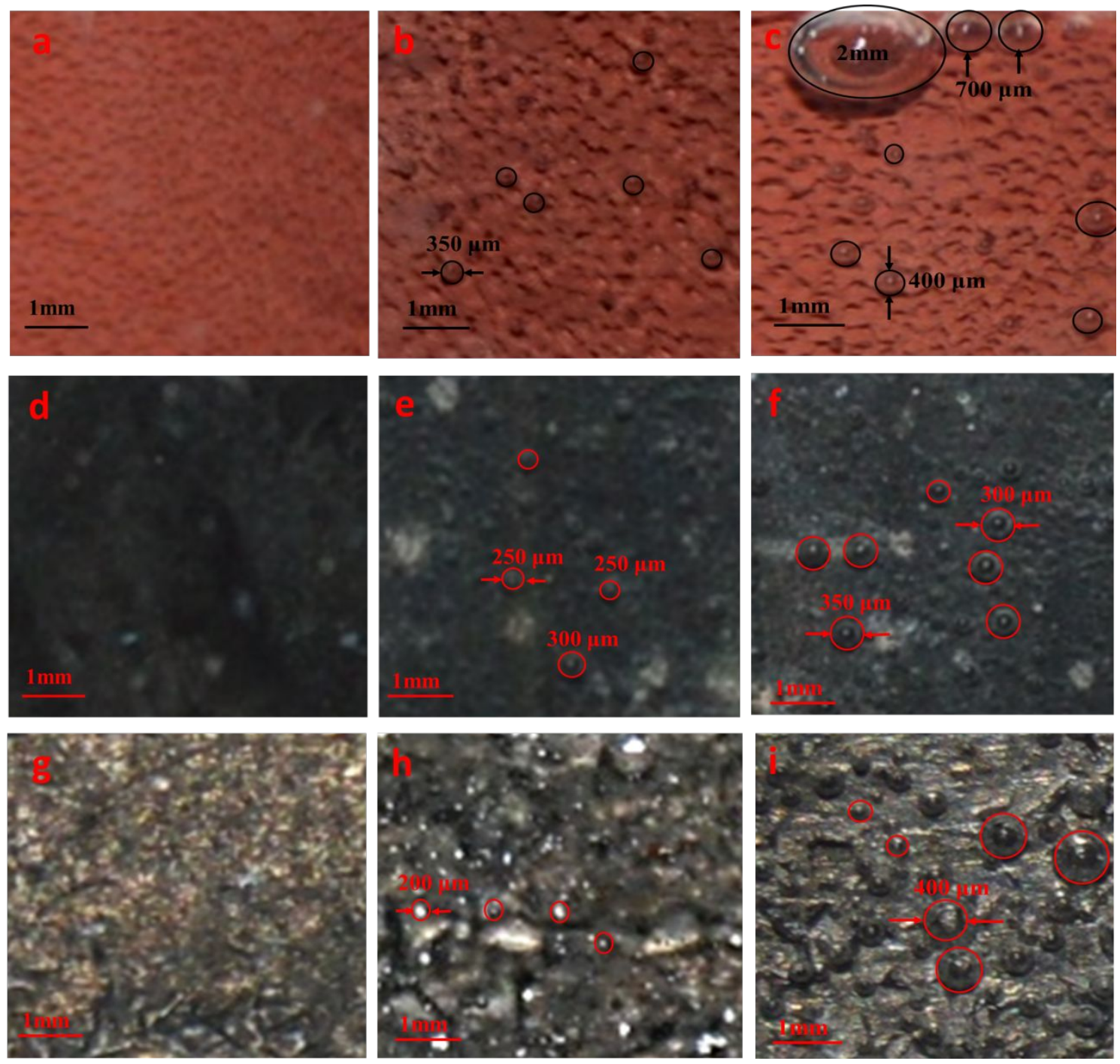

Figure S14. Aerophobic behaviour of the control samples (a-c) bare Cuf, (d-f) $\mathrm{Ni}_{5} \mathrm{P}_{4} / \mathrm{Cu},(g-i)$ $\mathrm{Ni}_{5} \mathrm{P}_{4} / \mathrm{GP}$ 

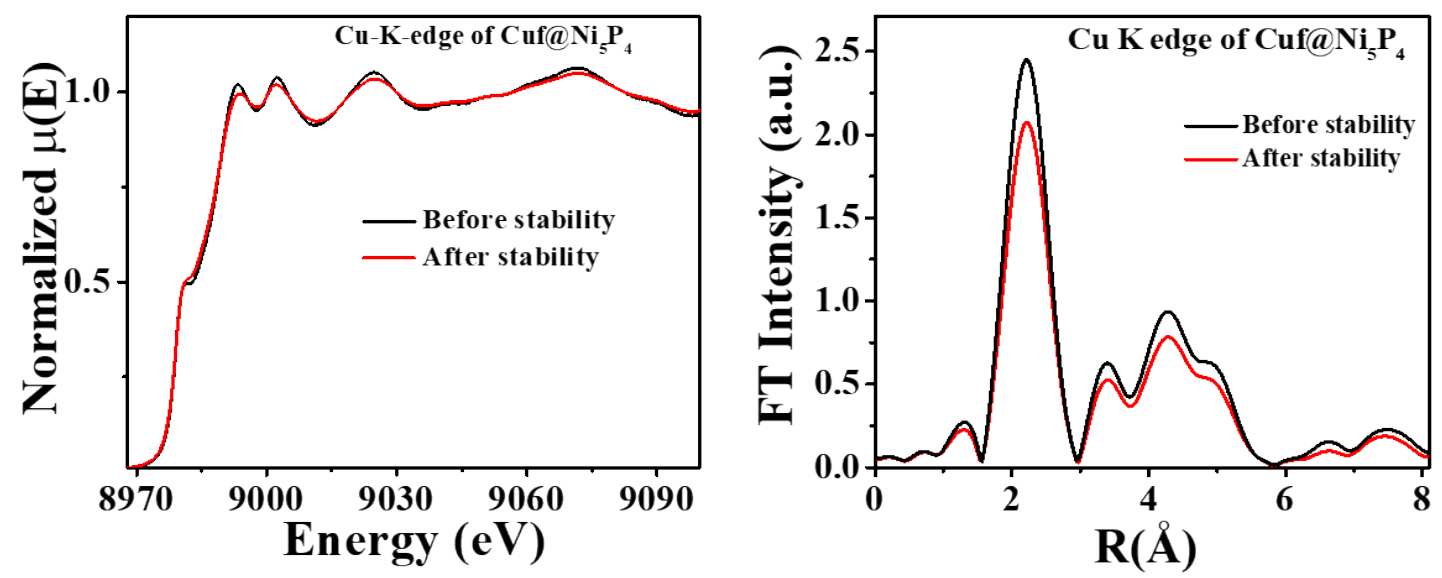

Figure S15. (c) XANES spectra and (d) FT-EXFAS signal of Cuf@Ni $\mathrm{P}_{4}$ for before and after stability at Cu K-edge. 

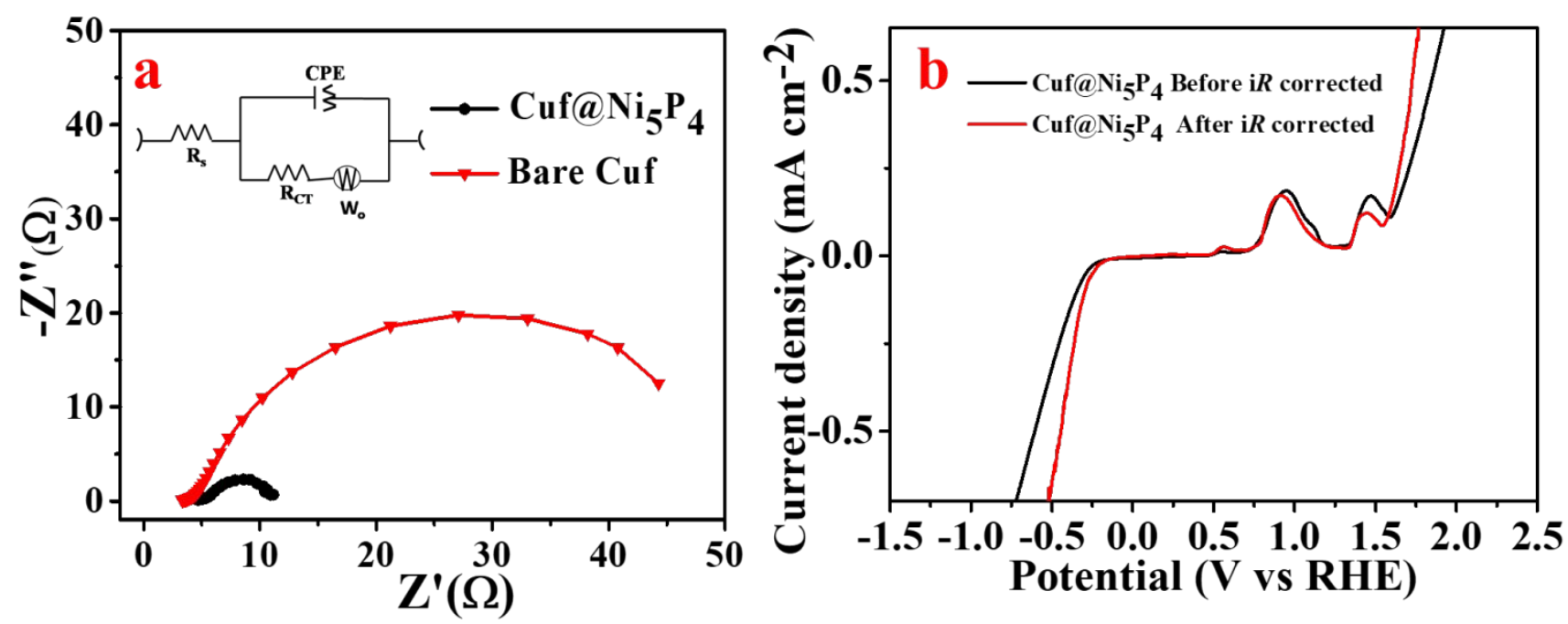

Figure S16. (a) Nyquist plot of bare Cuf and Cuf@ $\mathrm{Ni}_{5} \mathrm{P}_{4}$ at $1.58 \mathrm{~V}$ (vs RHE), (b) LSV curve full scan before and after iR compensation. 


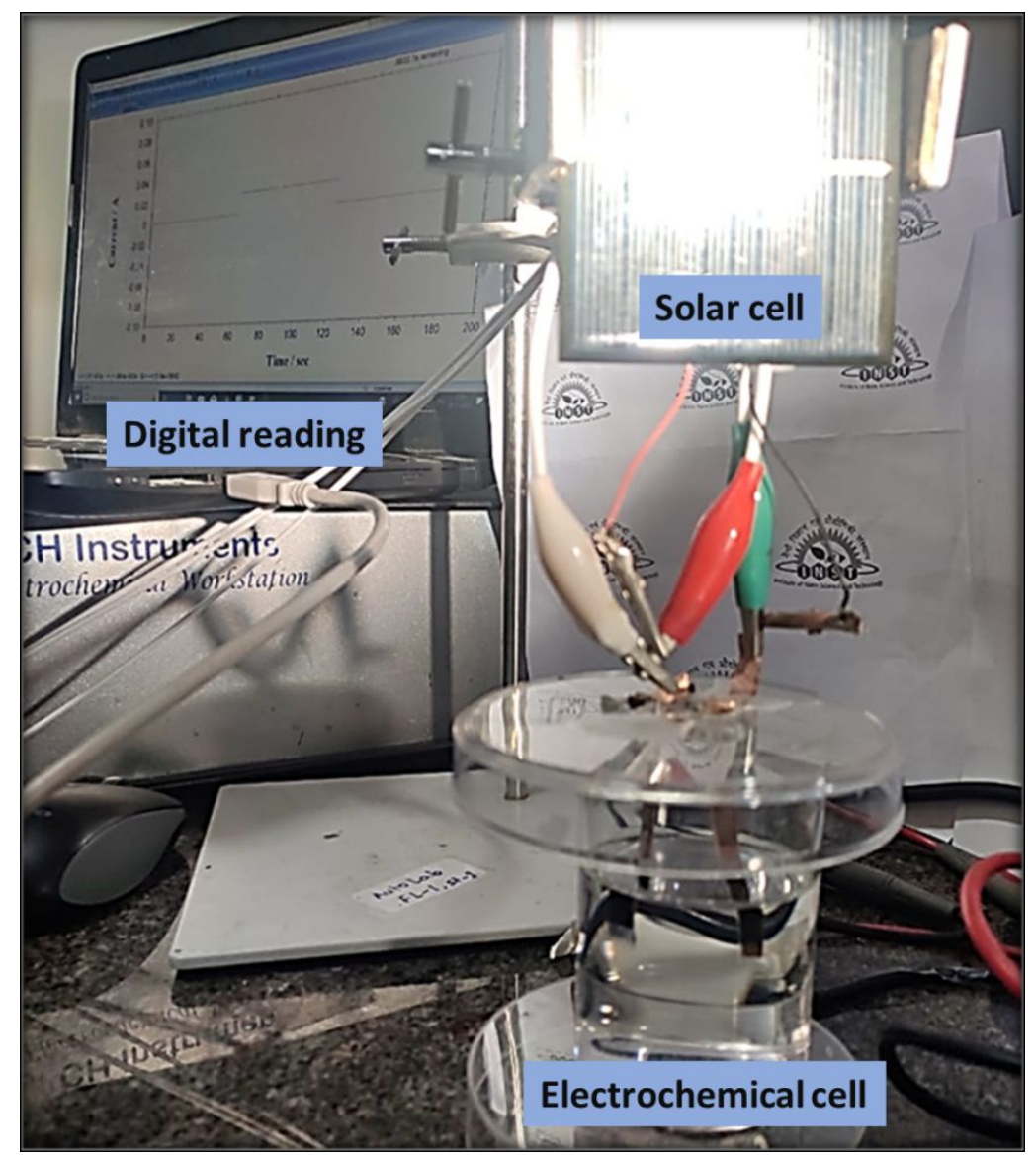

Figure S17. Digital image of Solar cell-water electrolysis hybrid with electrochemical reading. 
Table S1. Comparison for OER onset potentials of Cuf@ $\mathrm{Ni}_{5} \mathrm{P}_{4}$ with recently developed catalysts

\begin{tabular}{|c|c|c|}
\hline Catalyst & $\eta_{\text {onset }}(\mathrm{V}$ vs RHE) & References \\
\hline $\mathrm{CoP} / \mathrm{C}$ & 1.5 & ACS Catal. 2015, 5, 4066-4074 \\
\hline $\mathrm{Ni}_{2} \mathrm{P}-\mathrm{NW}$ & 1.54 & $\begin{array}{c}\text { Chem. Commun., 2015, 51, 11626-- } \\
11629\end{array}$ \\
\hline $\mathrm{Ni}_{2} \mathrm{P}-\mathrm{NP}$ & 1.61 & $\begin{array}{l}\text { Energy Environ. Sci., 2015,8, 2347- } \\
2351\end{array}$ \\
\hline $\mathrm{N}-\mathrm{CG}-\mathrm{CoO}$ & 1.55 & Energy Environ. Sci., 2014, 7, 609-616 \\
\hline IPNT & 1.48 & Chem. Eur. J. 2015, 21, $18062-18067$ \\
\hline Ni-Co Oxide & 1.49 & ACS Nano, 2014, 8, 9, 9518-9523 \\
\hline $\mathrm{LiCoO}_{2}$ & 1.55 & $\begin{array}{c}\text { Nature Communications volume 5, } \\
\text { Article number: } 3949 \text { (2014) }\end{array}$ \\
\hline $\mathrm{Au} @ \mathrm{Co}_{3} \mathrm{O}_{4}$ & 1.53 & Adv. Mater. 2014, 26, 23, 3950-3955 \\
\hline CoMn LDH & 1.5 & $\begin{array}{l}\text { J. Am. Chem. Soc. 2014, 136, } \\
\text { 16481-16484 }\end{array}$ \\
\hline $\mathrm{Ni}_{\mathrm{x}} \mathrm{Co}_{3-\mathrm{x}} \mathrm{O}_{4}$ & 1.53 & Adv. Mater. 2010, 22, 1926-1929 \\
\hline $\mathrm{Mn}_{0.1} \mathrm{Ni}$ & 1.59 & Adv. Funct. Mater. 2014, 25, 3, 393-399 \\
\hline NiFe-LDH & 1.45 & J. Am. Chem. Soc. 2013, 135, 8452-8455 \\
\hline FeCoNi-ATNs/NF & 1.46 & Adv. Energy Mater. 2019, 1901312 \\
\hline $\mathrm{NCN} / \mathrm{CC}$ & 1.48 & J. Mater. Chem. $A, 2018,6,4466-4476$ \\
\hline $\mathrm{Cuf@Ni} \mathrm{Ni}_{5} \mathrm{P}_{4}$ & 1.43 & This Work \\
\hline
\end{tabular}

NW (Nanowires), NP (Nanoparticles), N-CG-CoO (3D crumpled graphene-cobalt oxide), IPNT (iron phosphide nanotube), LDH (Layered double hydroxide), ATNs (Atomically thin nanosheets), NF (Nickel Foam), NCN/CC (nickel-cobalt nitride on carbon cloth), 
Table S2. Electrochemical activities of the catalytic electrodes for overall water splitting.

\begin{tabular}{|c|c|c|c|c|}
\hline Catalyst & $\begin{array}{c}\mathbf{\eta} @ 10 \\
\mathbf{m A ~} \mathbf{~ c m}^{-2} \\
\end{array}$ & Electrolyte & $\begin{array}{c}\text { Stability } \\
\text { (hr) }\end{array}$ & Reference \\
\hline $\begin{array}{l}\mathrm{EG} / \mathrm{Co}_{0.85} \mathrm{Se} / \mathrm{NiFe}- \\
\mathrm{LDH}\end{array}$ & $1.67 \mathrm{~V}$ & $1 \mathrm{M} \mathrm{KOH}$ & 10 & $\begin{array}{l}\text { Energy Environ. Sci., 2016, 9, } \\
478-483\end{array}$ \\
\hline $\mathrm{NiCO}_{2} \mathrm{O}_{4}$ & $1.65 \mathrm{~V}$ & $1 \mathrm{M} \mathrm{NaOH}$ & 36 & $\begin{array}{l}\text { Angew. Chem. Int. Ed. 2016, 55, } \\
\text { 6290-6294 }\end{array}$ \\
\hline $\mathrm{CP} / \mathrm{CTs} / \mathrm{Co}-\mathrm{S}$ & $1.743 \mathrm{~V}$ & $1 \mathrm{M} \mathrm{KOH}$ & 1.94 & ACS Nano 2016, 10, 2342-2348 \\
\hline $\mathrm{NiS} / \mathrm{NF}$ & $1.64 \mathrm{~V}$ & $1 \mathrm{M} \mathrm{KOH}$ & 35 & $\begin{array}{l}\text { Chem. Commun., 2016, 521,1486- } \\
1489\end{array}$ \\
\hline $\mathrm{Ni} / \mathrm{Ni}_{8} \mathrm{P}_{3}$ & $1.61 \mathrm{~V}$ & $1 \mathrm{M} \mathrm{KOH}$ & 4.1 & $\begin{array}{l}\text { Adv. Funct. Mater. 2016, 26, } \\
\text { 3314-3323 }\end{array}$ \\
\hline $\mathrm{PNC} / \mathrm{Co}$ & $1.64 \mathrm{~V}$ & $1 \mathrm{M} \mathrm{KOH}$ & 9.7 & $\begin{array}{l}\text { J. Mater. Chem. A, 2016, 4, 3204- } \\
3209\end{array}$ \\
\hline $\mathrm{NiFe} \mathrm{LDH} / \mathrm{NF}$ & $1.7 \mathrm{~V}$ & $1 \mathrm{M} \mathrm{NaOH}$ & & Science, 2014, 345, 1593 \\
\hline Ni@NC & $1.81 \mathrm{~V}$ & $1 \mathrm{M} \mathrm{KOH}$ & 10 & $\begin{array}{l}\text { Adv. Energy Mater. 2015, 5, } \\
1401660\end{array}$ \\
\hline $\mathrm{Ni}_{2 / 3} \mathrm{Fe}_{1 / 3}-\mathrm{rGO}$ & $2.02 \mathrm{~V}$ & $1 \mathrm{M} \mathrm{KOH}$ & 10 & ACS Nano,2015,92,1977-1984 \\
\hline $\mathrm{NiMoP}_{2} / \mathrm{CC}$ & $1.67 \mathrm{~V}$ & $1 \mathrm{M} \mathrm{KOH}$ & 24 & $\begin{array}{l}\text { J. Mater. Chem. A, 2017, 5, 7191- } \\
7199\end{array}$ \\
\hline NiCoP & $1.77 \mathrm{~V}$ & $1 \mathrm{M} \mathrm{KOH}$ & 20 & Nano Res. 2016, 9(8): 2251-2259 \\
\hline $\mathrm{Ni}_{8} \mathrm{P}_{3} / \mathrm{NF}$ & $1.79 \mathrm{~V}$ & $1 \mathrm{M} \mathrm{KOH}$ & 22.2 & $\begin{array}{l}\text { ACS Appl. Mater. Interfaces } 2016 \text {, } \\
8,27850-27858\end{array}$ \\
\hline $\mathrm{C} @ \mathrm{Ni}_{8} \mathrm{P}_{3}$ & $1.65 \mathrm{~V}$ & $1 \mathrm{M} \mathrm{KOH}$ & 22.2 & $\begin{array}{l}\text { ACS Appl. Mater. Interfaces 2016, } \\
8,27850-27858\end{array}$ \\
\hline $\mathrm{Ni}_{5} \mathrm{P}_{4} / \mathrm{Ni}$ foil & $1.69 \mathrm{~V}$ & $1 \mathrm{M} \mathrm{KOH}$ & - & Angew. Chem., 2015, 127, 12538 \\
\hline $\mathrm{Ni}_{2} \mathrm{P} / \mathrm{NF}$ & $1.63 \mathrm{~V}$ & $1 \mathrm{M} \mathrm{KOH}$ & 10 & $\begin{array}{l}\text { Energy Environ. Sci., 2015, 8, } \\
2347\end{array}$ \\
\hline $\mathrm{CoP}-\mathrm{Cu}$ foil & $1.65 \mathrm{~V}$ & $1 \mathrm{M} \mathrm{KOH}$ & 24 & $\begin{array}{l}\text { Angew. Chem., Int. Ed., } \\
\text { 2015, 54, } 6251\end{array}$ \\
\hline $\mathrm{Co}_{\mathrm{x}} \mathrm{PO}_{4} / \mathrm{CoP}-\mathrm{Au}$ & $1.91 \mathrm{~V}$ & $1 \mathrm{M} \mathrm{KOH}$ & - & Adv. Mater., 2015, 27, 3175. \\
\hline $\begin{array}{l}\mathrm{CoP} / \mathrm{GO}-400 \\
\mathrm{GCE}\end{array}$ & $1.70 \mathrm{~V}$ & $1 \mathrm{M} \mathrm{KOH}$ & 24 & Chem. Sci., 2016, 7, 1690 \\
\hline $\begin{array}{l}\mathrm{Co}_{0.13} \mathrm{Ni}_{0.87} \mathrm{Se}_{2} / \mathrm{Ti} \\
\text { plate }\end{array}$ & $1.62 \mathrm{~V}$ & $1 \mathrm{M} \mathrm{KOH}$ & 10 & Nanoscale, 2016, 8, 3911. \\
\hline $\mathrm{NiFeP} / \mathrm{NF}$ & $1.67 \mathrm{~V}$ & $1 \mathrm{M} \mathrm{KOH}$ & 40 & $\begin{array}{l}\text { ACS Appl. Mater. Interfaces, } \\
\text { 2017, 9, 31, 26134-26142 }\end{array}$ \\
\hline $\mathrm{NCN} / \mathrm{CC}$ & $1.56 \mathrm{~V}$ & $1 \mathrm{M} \mathrm{KOH}$ & 250 & J. Mater. Chem. $A, 2018,6,4466-4476$ \\
\hline $\mathrm{Cuf} @ \mathrm{Ni}_{5} \mathrm{P}_{4}$ & $1.66 \mathrm{~V}$ & $1 \mathrm{M} \mathrm{KOH}$ & 150 & This work \\
\hline
\end{tabular}

NF-Nickel foam, GCE- Glassy carbon electrode, ONPPGC/OCC -Nitrogen, phosphorus and oxygen tri-doped porous graphite carbon@oxidized carbon cloth, LDH- Layered double hydroxide, rGO- Reduced graphene oxide, $\mathrm{CP} / \mathrm{CTs} / \mathrm{Co}-\mathrm{S}$ - carbon paper/carbon tubes/cobaltsulfide sheets, EG- exfoliated graphene, PNC- porous N-rich carbon, NC- Nitrogen doped carbon, CC- Carbon cloth. 
Table S3. OER Potential of the electrodes at different current densities

\begin{tabular}{|c|c|c|c|c|c|c|c|}
\hline & Catalyst & n@Onset & $\begin{array}{l}\mathbf{n @ 2 0} \\
\mathbf{m A} \\
\mathrm{cm}^{-2}\end{array}$ & $\begin{array}{l}\text { 1@50 } \\
\mathbf{m A} \\
\mathrm{cm}^{-2}\end{array}$ & $\begin{array}{l}\text { ᄁ@100 } \\
\text { mA } \\
\mathrm{cm}^{-2}\end{array}$ & $\begin{array}{l}\eta @ 500 \\
\mathbf{m A} \\
\mathrm{cm}^{-2}\end{array}$ & $\begin{array}{l}\text { ү@700 } \\
\text { mA cm }\end{array}$ \\
\hline \multirow{5}{*}{ OER } & $\mathrm{Cuf} @ \mathrm{Ni}_{5} \mathrm{P}_{4}$ & $1.42 \mathrm{~V}$ & $1.44 \mathrm{~V}$ & $1.52 \mathrm{~V}$ & $1.56 \mathrm{~V}$ & $1.72 \mathrm{~V}$ & $1.77 \mathrm{~V}$ \\
\hline & $\mathrm{Ni}_{5} \mathrm{P}_{4} / \mathrm{Cu}$ & $1.52 \mathrm{~V}$ & $1.56 \mathrm{~V}$ & $1.60 \mathrm{~V}$ & $1.64 \mathrm{~V}$ & - & - \\
\hline & $\mathrm{Ni}_{5} \mathrm{P}_{4} / \mathrm{GP}$ & $1.57 \mathrm{~V}$ & - & $1.68 \mathrm{~V}$ & $1.82 \mathrm{~V}$ & - & - \\
\hline & Cuf & $1.68 \mathrm{~V}$ & - & $1.80 \mathrm{~V}$ & $1.94 \mathrm{~V}$ & - & - \\
\hline & $\mathrm{RuO}_{2}$ & $1.43 \mathrm{~V}$ & $1.45 \mathrm{~V}$ & $1.50 \mathrm{~V}$ & $1.55 \mathrm{~V}$ & $1.85 \mathrm{~V}$ & $2.02 \mathrm{~V}$ \\
\hline
\end{tabular}


Table S4. HER Potential of the electrodes at different current densities

\begin{tabular}{|c|c|c|c|c|c|c|c|}
\hline & Catalyst & 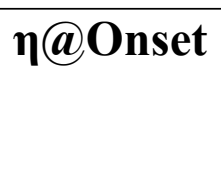 & $\begin{array}{l}\mathbf{\eta} @ \mathbf{2 0} \\
\mathbf{m A ~ c m} \mathbf{c m}^{-2}\end{array}$ & 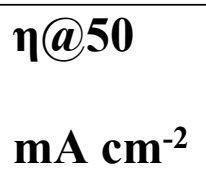 & 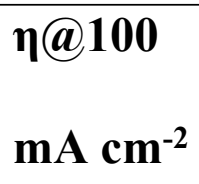 & 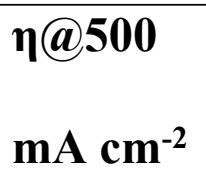 & $\begin{array}{l}\eta @ 700 \mathrm{~mA} \\
\mathrm{~cm}^{-2}\end{array}$ \\
\hline \multirow{5}{*}{ HER } & Cuf@ $@ \mathrm{Ni}_{5} \mathrm{P}_{4}$ & $53 \mathrm{mV}$ & $192 \mathrm{mV}$ & $247 \mathrm{mV}$ & $298 \mathrm{mV}$ & $446 \mathrm{mV}$ & $514 \mathrm{mV}$ \\
\hline & $\mathrm{Ni}_{5} \mathrm{P}_{4} / \mathrm{Cu}$ & $115 \mathrm{mV}$ & $232 \mathrm{mV}$ & $298 \mathrm{mV}$ & $360 \mathrm{mV}$ & - & - \\
\hline & $\mathrm{Ni}_{5} \mathrm{P}_{4} / \mathrm{GP}$ & $147 \mathrm{mV}$ & $298 \mathrm{mV}$ & $403 \mathrm{mV}$ & $528 \mathrm{mV}$ & - & - \\
\hline & Cuf & $224 \mathrm{mV}$ & $474 \mathrm{mV}$ & $622 \mathrm{mV}$ & $798 \mathrm{mV}$ & - & - \\
\hline & $\mathrm{Pt} / \mathrm{C}$ & $13 \mathrm{mV}$ & $46 \mathrm{mV}$ & $83 \mathrm{mV}$ & $122 \mathrm{mV}$ & - & - \\
\hline
\end{tabular}


Supporting video 1: Supporting video shows the performance of water electrolysis cell when the electrochemical cell was hybridized with commercial solar cell upon illumination. 